\title{
INTERVENCIÓN EDUCATIVA SOBRE PARASITOSIS INTESTINAL EN PADRES DE FAMILIA DE NIÑOS DE 3 a 5 AÑOS.
}

\author{
EDUCATIONAL INTERVENTION ON INTESTINAL PARASITOSIS IN FAMILY PARENTS OF CHILDREN OF 3 - 5 \\ YEARS.
}

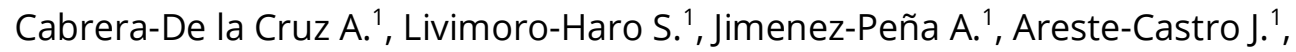 Sanchez-Salinas A. ${ }^{1}$, Checa-Braga S. ${ }^{1}$, Quintana-Cañari A. ${ }^{1}$, Magallanes-Sebastián M. $^{2}$
https://doi.org/10.52109/cyp2022324
${ }^{1}$ Laboratorio Clínico y Anatomía Patológica de la Universidad Nacional Mayor de San Marcos
2 Docente asociado de la Universidad Nacional Mayor de San Marcos, e-mail: mmagallaness@unmsm.edu.pe, https://orcid.org/0000-0001-6920-902X

\begin{tabular}{l} 
REGISTROS \\
\hline Recibido el \\
$16 / 10 / 2021$ \\
Aceptado el \\
$27 / 01 / 2022$ \\
Publicado el \\
$31 / 01 / 2022$ \\
CC 9 EY SA \\
PALABRAS CLAVE \\
\hline Parasitosis intestinal, \\
conocimientos, \\
actitudes, parasitosis \\
en niños, intervención \\
educativa. \\
KEYWORDS \\
\hline Intestinal parasitosis, \\
knowledge, attitudes, \\
parasitosis in children, \\
educational \\
intervention.
\end{tabular}

\section{RESUMEN}

La parasitosis intestinal es una enfermedad contagiosa causada principalmente por lombrices o gusanos que se alojan en los intestinos, siendo la población infantil la más afectada. El objetivo del estudio fue evaluar los conocimientos y actitudes en padres de familia sobre parasitosis intestinal en niños de 3 - 5 años de la I.E. 412 Hoja Redonda, Chincha - Ica. Se empleó un diseño Preexperimental de enfoque cuantitativo, descriptivo y transversal. Se realizaron 7 sesiones virtuales y la evaluación de los padres de familia mediante dos encuestas en dos fases. En la primera fase el 12\% de los 68 padres de familia encuestados respondieron acertadamente $(<50 \%$ de preguntas), $4 \%$ respondió (50\% de preguntas), 31\% respondió (entre el $50 \%$ y $75 \%$ de preguntas), 53\% respondió (entre el $75 \%$ y $<100 \%$ de preguntas) y $0 \%$ respondió (100\% de preguntas). En la segunda fase $2 \%$ respondieron acertadamente ( $<50 \%$ de preguntas), $6 \%$ respondió $(50 \%$ de preguntas), 39\% respondió (entre el 50\% y 75\% de preguntas), $49 \%$ respondió (entre el $75 \%$ y $<100 \%$ de preguntas) y $4 \%$ respondió el $100 \%$ de preguntas. Conclusión. El trabajo realizado logró mejorar el conocimiento y actitudes de los padres de familia sobre la parasitosis intestinal en niños. 
of questions) and $4 \%$ answered $100 \%$ of questions. Conclusion: The work carried out managed to improve the knowledge and attitudes of parents about intestinal parasitosis in children.

\section{INTRODUCCIÓN}

La parasitosis intestinal es un problema que aqueja a la cuarta parte de la población mundial. Sin embargo, los países en vías de desarrollo son los que más lo sufren y el Perú no es la excepción, pues se ubica dentro de los cinco países con mayor prevalencia en América Latina. Según el INS (2018), el 40\% de niños entre 2 y 5 años presentan parasitosis intestinal, siendo Loreto, San Martín, Ucayali, Amazonas y Pasco los departamentos con mayor prevalencia, sin embargo, también presentan una positiva reducción porcentual anual de niños infectados en los últimos 10 años. Mientras que departamentos como Lima, Callao, Ica y otros mantuvieron un porcentaje de parasitosis aproximadamente constante durante el 2010 y 2017, por lo que resulta beneficioso abordar dicha problemática desde la educación y prevención en poblaciones afectadas, con la intención de generar conocimiento y sensibilización acerca de la parasitosis intestinal, siendo de gran ayudad que se realice a través de una institución educativa promotora que facilite las respectivas coordinaciones para la realización de las charlas virtuales, ya que por la coyuntura actual, no se podría realizar presencialmente.

Estos problemas de salud pública tienen mayor prevalencia en países subdesarrollados, sobre todo en zonas rurales y urbano-marginales por las condiciones propicias para su proliferación y las malas condiciones sanitarias, teniendo como principales afectados a los niños, perjudicando su salud, causando deficiencia de aprendizaje y función cognitiva. Por lo general, esta enfermedad pasa desapercibida por ser asintomática, pero es un factor de morbilidad importante cuando va de la mano con la desnutrición, siendo los niños en edad escolar la población más vulnerable por su falta de conocimientos y malos hábitos que pueden tener de lavado de alimentos, ingesta de agua contaminada, mal lavado de manos, etc.

En el Perú aún no hay estudios de prevalencia de parasitosis intestinal a nivel nacional, sin embargo, la Organización Panamericana de la Salud realizó un estudio en el 2013 basándose en modelos estadísticos y se estimó que el Perú tendría una prevalencia de parasitosis de $28,6 \%$ en población escolar.

La presente investigación busca brindar información a los padres de familia de la I.E. 412 Hoja Redonda, Chincha - Ica, acerca de la parasitosis intestinal en los niños. Se realizaron a través de plataformas virtuales sobre este tema a cargo de los integrantes del equipo y profesionales invitados como tecnólogos médicos, nutricionistas, debido al distanciamiento social en tiempos de pandemia. Para medir el nivel de conocimientos de los padres de familia sobre parasitosis intestinal en los niños, se realizó un pre test y un post test que permitió al equipo ver el impacto de la investigación en la comunidad a la cual nos dirigimos. 
En esta investigación se cumplió con 3 de los objetivos de desarrollo sostenible propuestos por las Naciones Unidas, como son: mejorar la salud y bienestar de nuestra comunidad, mejorar la educación buscando mejorar su calidad de aprendizaje y con ello contribuir a una reducción de las desigualdades a nivel nacional.

\section{MATERIALES Y MÉTODOS}

Estudio descriptivo realizado a padres de familia de niños de 3 - 5 años de la I.E. 412 Hoja Redonda, Chincha - Ica, ciudad ubicada a 200 km al sur de Lima-Perú. En la encuesta se recolectó datos generales del padre de familia, nivel de conocimiento sobre parasitosis intestinal y actitud de medidas de prevención de la parasitosis. Los datos obtenidos fueron recolectados en Microsoft Excel y presentados en tablas y gráficos.

\section{RESULTADOS}

Las preguntas fueron divididas en 2 grupos: conocimientos y actitudes. El grupo de preguntas sobre conocimientos de parasitosis intestinal, fueron agrupadas en secciones, la primera con los aspectos conceptuales sobre la parasitosis, en donde se observó un incremento en los aciertos de las preguntas en las post-charlas. La segunda sección estuvo relacionada con las manifestaciones clínicas, indicando que, en un primer momento, pre-charlas, hubo un gran porcentaje $(60 \%)$ de los padres de familia que respondieron correctamente en la mayoría de las preguntas, llegando a una calificación superior a 17, indicando así un gran conocimiento en este tema. En los cuestionarios post-charlas, se mostraron mejoras en todo el grupo de estudio. La tercera sección de preguntas estuvo relacionada con las vías de transmisión de la parasitosis en donde un 60\% de padres respondieron correctamente más del $75 \%$ de las preguntas, mejorando este en el segundo momento post inducción de las charlas educativas. Finalmente, la cuarta sección fue sobre las medidas de prevención de la parasitosis intestinal, en la cual se observó un mayor incremento de respuestas correctas, considerando así que las charlas educativas sobre la parasitosis intestinal tuvieron un efecto positivo en los padres de familia y en los niños.

Tabla 1

Cuestionario de la Fase I

\begin{tabular}{|c|c|}
\hline Pregunta & $\%$ \\
\hline A. ¿Cuántas personas NO respondieron correctamente todas las preguntas? & $0 \%$ \\
\hline $\begin{array}{l}\text { B. ¿Cuántas personas respondieron menos del 50\% de preguntas } \\
\text { correctamente? }\end{array}$ & $12 \%$ \\
\hline
\end{tabular}


C. ¿Cuántas personas respondieron el 50\% de preguntas correctamente?

D. ¿Cuántas personas respondieron mayor o igual del 50\% y menos del 75\% de preguntas correctamente?

E. ¿Cuántas personas respondieron mayor o igual del 75\% y menos del 100\% de preguntas correctamente?

F. ¿Cuántas personas respondieron correctamente a todas las preguntas?

Figura 1

\section{Nivel de conocimientos Fase I}

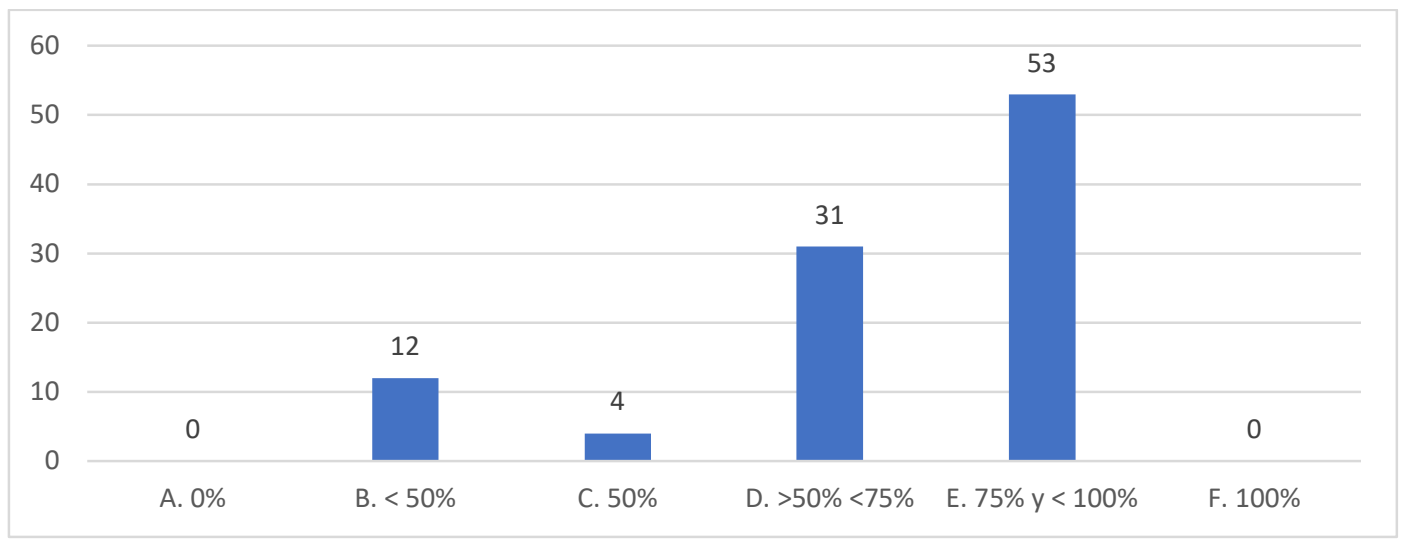

Figura 2

\section{Nivel de conocimientos Fase I}



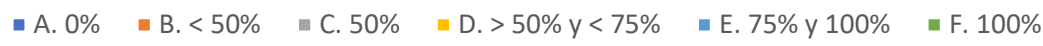


Figura 3

Nivel de conocimientos Fase II

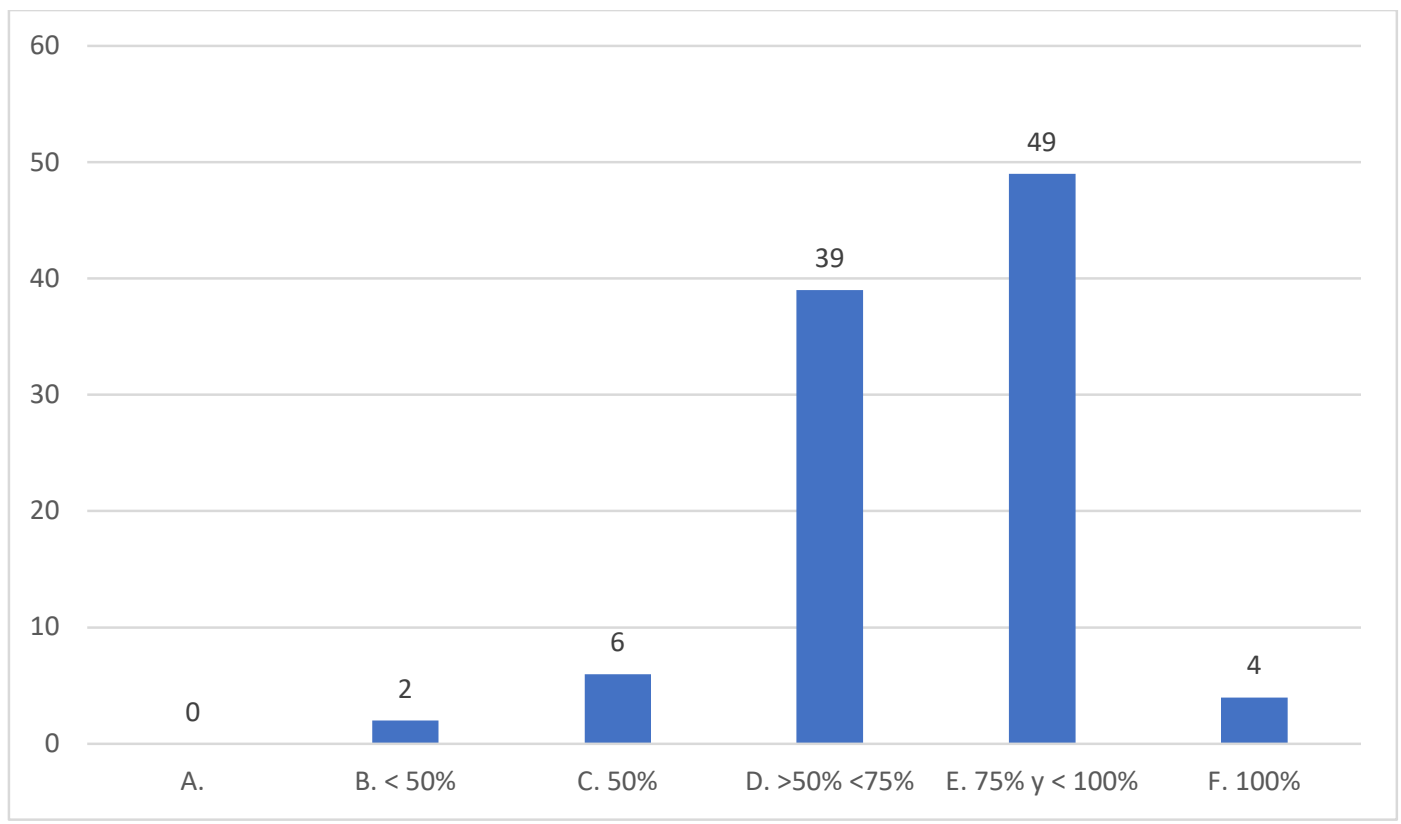

Figura 4

Nivel de conocimientos Fase II

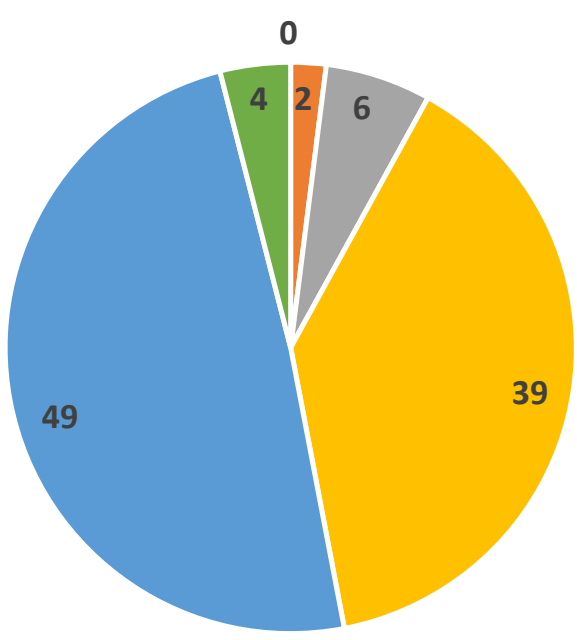

- A. $0 \%-$ B. $<50 \% \quad-$ C. $50 \% \quad-$ D. $>50 \%$ y $<75 \% \quad$ - E. $75 \%$ y $100 \% \quad-$ F. $100 \%$

El grupo de preguntas sobre actitudes de los padres de familia también se subdividieron en 3 secciones: higiene corporal, higiene y salud bucal e higiene ambiental, siendo un total de 17 preguntas. Cada respuesta dada por el padre de 
familia obtuvo un puntaje, si está totalmente de acuerdo equivale a 5 puntos; si está de acuerdo suma 4 puntos; si está indeciso ,3 puntos; en desacuerdo, 2 puntos y si está totalmente en desacuerdo solo 1 punto. La suma total de las 17 preguntas planteadas en cuestionario equivale a una actitud frente al rango establecido, si sumó de 17 a 51 puntos, se le considera con una actitud negativa y una actitud positiva si sumó de 51 a 85 puntos.

En el cuestionario pre-charlas, en total 68 personas respondieron ante lo cual se observó que 64 personas tuvieron una actitud positiva y 4 personas una actitud negativa. Después de realizar las 7 charlas virtuales a los padres de familia se aplicó el mismo cuestionario ante lo cual solo participaron 49 personas en donde se observó que 46 personas tuvieron una actitud positiva frente a 3 personas con actitud negativa.

Figura 5

Frecuencia de actitudes

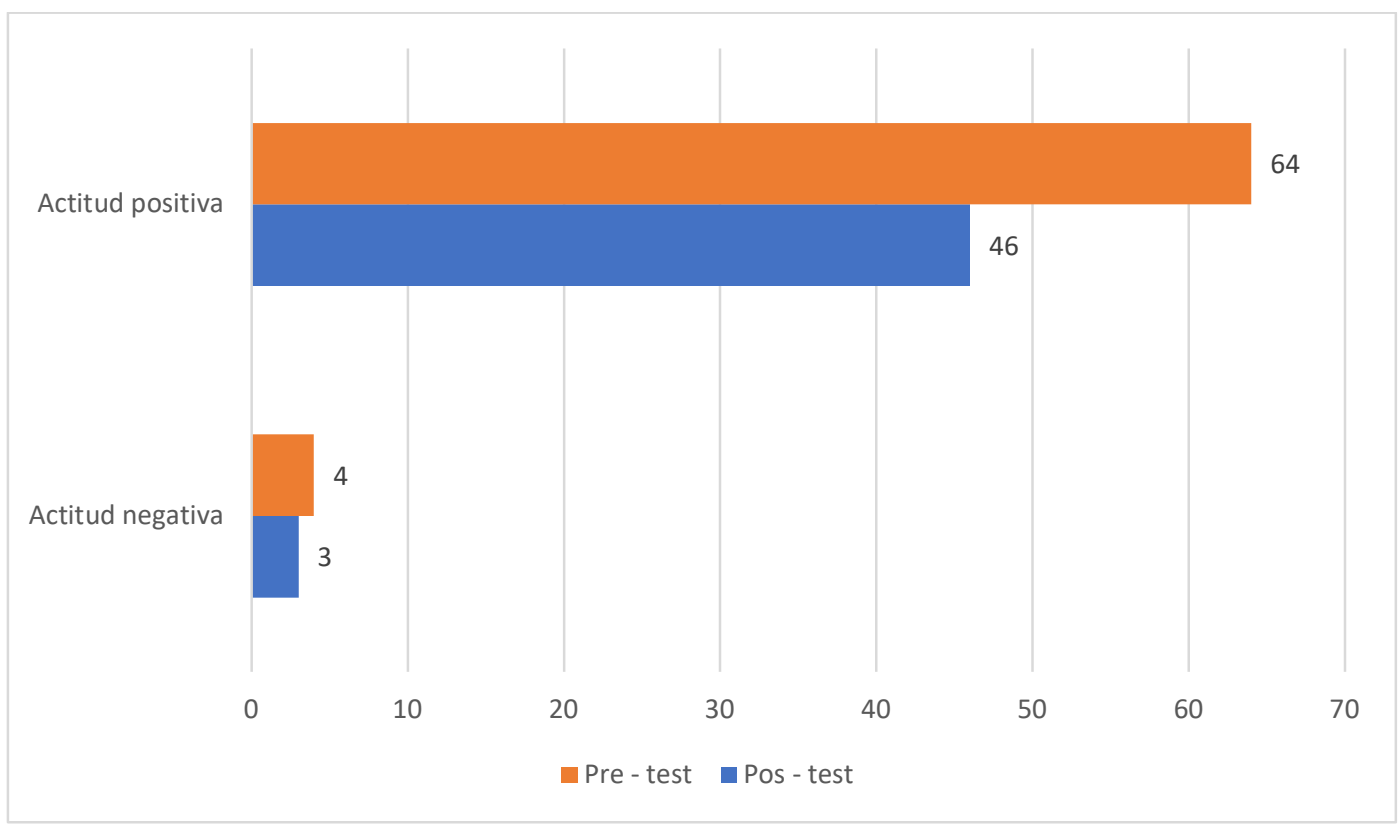

\section{DISCUSIÓN}

La parasitosis infantil es uno de los más grandes problemas de salud pública, su impacto repercute en el desarrollo, crecimiento, aprendizaje y comportamiento, siendo los niños menores de 5 años la población más vulnerable. Es por ello, que en la presente investigación se tuvo como objetivo general; evaluar los conocimientos y actitudes en padres de familia sobre parasitosis intestinal en niños de 3 a 5 años de la IE 412 Hoja Redonda, Chincha - Ica. La herramienta que se aplicó fue un cuestionario tomado en 2 momentos, previo y al término de las charlas. Al analizar los datos obtenidos en la investigación se encontró que de los 68 padres que realizaron la primera encuesta, 64 tienen actitudes positivas sobre las premisas 
dadas acerca de la higiene corporal, de cavidad y ambiental en relación con la parasitosis infantil, mientras que 4 de los 68 padres de familia presentan actitud negativa. En cuanto a nivel de conocimiento en el primer cuestionario se encontró que el $0 \%, 53 \%, 31 \%, 4 \%, 12 \%$ y $0 \%$ respondieron correctamente del total de preguntas un $100 \%$, mayor o igual del $75 \%$ y menos del $100 \%$, mayor o igual del $50 \%$ y menos del $75 \%, 50 \%$, menos del $50 \%$ y $0 \%$ respectivamente, mientras que en el segundo cuestionario se encontró que el 4\%, 49\%, 39\%, 6\%, 2\% y 0\% respondieron correctamente del total de preguntas un $100 \%$, mayor o igual del $75 \%$ y menos del $100 \%$, mayor o igual del $50 \%$ y menos del $75 \%$, 50\%, menos del $50 \%$ y $0 \%$ respectivamente.

Resultados similares encontraron Flores C, Seminario V (1) que determinó en su población con respecto a las actitudes para con la parasitosis intestinal un 97\% tenía una actitud positiva y $3 \%$ una actitud negativa y con respecto al conocimiento un $16 \%$ tenía un buen conocimiento, $67 \%$ un conocimiento regular y un $18 \%$ un conocimiento deficiente, esto también se visualizó en el estudio de Peña M. en donde tanto en conocimiento como en actitud los valores fueron mayores al 50\%, resultados opuestos a los de Tuesta $M$. que determinó como resultado de su estudio que, del total, un $60 \%$ tenía un conocimiento deficiente mientras solamente el $40 \%$ si conocía sobre parasitosis intestinal.

Al igual que en el estudio realizado por Nakandakari (3), uno de los principales factores que influyó en los resultados, fue que la población en su gran mayoría, vivían en condiciones rurales.

A través del abordaje multidisciplinario en el desarrollo de las charlas se pudo brindar una información más completa a los padres de familia, se contó con nutricionistas, tecnólogos médicos en laboratorio clínico y microbiólogos, que en conjunto con los internos del equipo de "Cazadores de parásitos" permitieron la presentación de variadas propuestas para mejorar la calidad de vida y el rendimiento escolar en los niños de 3 a 5 años de la IE 412 Hoja Redonda, Chincha - Ica.

\section{CONCLUSIONES}

Los conocimientos que tienen los padres de familia sobre la parasitosis intestinal en niños de 3 - 5 años de la I.E. 412 Hoja Redonda, Chincha - Ica en base a los resultados obtenidos en nuestro proyecto evidencian un buen conocimiento, a la vez, se encontró una participación activa de los padres de familia en las diferentes charlas brindadas lo cual es un indicador de la responsabilidad, preocupación y actitud reflexiva con respecto a la parasitosis intestinal en niños.

Con respecto a las encuestas realizadas, se mostró una gran mejoría en las respuestas brindadas por los padres de familia posterior a las charlas educativas que se impartieron por parte de los docentes especializados en temas sobre la parasitosis intestinal. En la sección de conocimientos relacionados a los aspectos 
conceptuales sobre la parasitosis intestinal, hubo una leve mejoría en las respuestas acertadas; sin embargo, podemos conjeturar la existencia aún de ciertas dudas en este subtema.

En el conocimiento de las manifestaciones clínicas de la parasitosis hubo una mejoría notoria, esto influenciado por la motivación y entusiasmo por parte de todos los participantes, llegando a encontrarse así hasta un 100\% de respuestas correctas en una pregunta e incrementándose las respuestas asertivas en las demás preguntas, de esta manera se puede concluir que se logró el objetivo planteado en esta sección. De la misma manera, en la sección de vías de transmisión de la parasitosis intestinal, hubo un incremento de respuestas acertadas en el segundo cuestionario, reconociendo que estos se pueden transmitir mediante la ropa interior y sábanas contaminadas, por tener las manos y uñas sucias llevándolas a la boca. En la sección sobre las medidas de prevención de la parasitosis intestinal en niños, en general, existe un buen nivel de conocimientos y se pudo constatar que hubo incremento en las respuestas acertadas para tres de las cinco preguntas planteadas en este ítem. Reconociendo así que es importante un correcto aseo de manos, el lavado de los alimentos antes de consumirlos, el tomar medidas preventivas para evitar la transmisión de parásitos recogiendo adecuadamente y desechando de forma segura las heces de las mascotas.

El presente proyecto ha pretendido realizar una propuesta de ApS a través de la divulgación científica de enfermedades parasitarias con la utilización de herramientas digitales en padres de familia de una institución educativa inicial, con la participación de estudiantes universitarios de la escuela de Tecnología Médica de la UNMSM. Los objetivos logrados sobre la divulgación de la parasitosis intestinal han sido los esperados. Asimismo, el proyecto visto desde una perspectiva de educación superior nos ha permitido desarrollar la metodología de enseñanza y aprendizaje, también nos ha llevado a conocer y reflexionar sobre la experiencia desarrollada en tiempos de pandemia. Esperamos a través de esta propuesta, incentivar futuros proyectos que consoliden la relación planteada entre la universidad y la comunidad, logrando de esta manera promover la formación integral de los futuros profesionales con un sentido de responsabilidad social.

\section{REFERENCIAS}

Flores Torres CP, Seminario Cieza V del C. Conocimientos, actitudes y prácticas de las madres para prevenir parasitosis intestinal en niños preescolares. Caserío Cruz del Médano, Mórrope- 2018. 2020; Disponible en: http://tesis.usat.edu.pe/handle/20.500.12423/2400

Moffa M, Cronk R, Fejfar D, Dancausse S, Padilla LA, Bartram J. A systematic scoping review of hygiene behaviors and environmental health conditions in institutional care settings for orphaned and abandoned children. Sci Total Environ. 25 de marzo de 2019; 658:1161-74. 
Nakandakari MD, De la Rosa DN, Beltrán-Fabián M. Enteroparasitosis en niños de una comunidad rural de Lima-Perú. Rev Medica Hered. abril de 2016;27(2):969.

Navarro JA, Villafranca RC, Alpízar M del CM, Hernández SVC, Piloto IZ. Reflexiones pertinentes sobre la parasitosis intestinal en los círculos infantiles. Rev Cuba Hig Epidemiol. 2017;55(1):34-43.

Sandoval P, Cristina M. Conocimientos, actitudes y prácticas sobre medidas preventivas de parasitosis intestinal en madres de niños menores de 5 años atendidos en Puesto de Salud Puente Internacional, diciembre 2017- abril 2018. Univ San Pedro [Internet]. 16 de mayo de 2019; Disponible en: http://repositorio.usanpedro.edu.pe//handle/USANPEDRO/11807

Tuesta M. Conocimiento de las madres sobre parasitosis intestinal en niños de 6 meses a 2 años que acudieron al control de crecimiento y desarrollo en el Centro de Salud Palo de Acero - Huánuco - 2015. Disponible en: https://cybertesis.unmsm.edu.pe/handle/20.500.12672/6276

Vidal-Anzardo M, Yagui Moscoso M, Beltrán Fabian M, Vidal-Anzardo M, Yagui Moscoso M, Beltrán Fabian M. Parasitosis intestinal: Helmintos. Prevalencia y análisis de la tendencia de los años 2010 a 2017 en el Perú. An Fac Med. marzo de 2020;81(1):26-32.

Zuta Arriola N, Rojas Salazar AO, Mori Paredes MA, Cajas Bravo V. Impacto de la educación sanitaria escolar, hacinamiento y parasitosis intestinal en niños preescolares. Comuni@cción. junio de 2019;10(1):47-56. 